\title{
Những yếu tố ảnh hưởng đến việc sử dụng Ethephon cho sầu riêng sau thu hoạch tại tỉnh Tiền Giang
}

\section{Factors determining the behavioral usage of the Ethephon for post- harvest durian in Tien Giang Province}

\author{
Bùi Thị Kim Hoàng ${ }^{1 *}$, Nguyễn Thị Mỹ Tiên ${ }^{1}$, Đặng Lê Khoa ${ }^{2}$ \\ ${ }^{1}$ Trường Đại học Nông Lâm Thành phố Hồ Chí Minh, Việt Nam \\ ${ }^{2}$ Trường Đại học Khoa học Tự nhiên - Đại học Quốc gia Thành phố Hồ Chí Minh, Việt Nam \\ *Tác giả liên hệ, Email: btkimhoang@ @cmuaf.edu.vn
}

\begin{tabular}{|c|c|}
\hline THÔNG TIN & TÓM TẮT \\
\hline $\begin{array}{l}\text { DOI: } 10.46223 / \mathrm{HCMCOUJS} \text {. } \\
\text { econ.vi.16.3.2000.2021 }\end{array}$ & $\begin{array}{l}\text { Bài báo này khảo sát những yếu tố ảnh hưởng đến việc sử } \\
\text { dụng Ethephon để giúp chín trái sầu riêng sau thu hoạch. Mô hình } \\
\text { nghiên cứu chủ yếu dựa trên lý thuyết hợp nhất về chấp nhận và }\end{array}$ \\
\hline Ngày nhận: 28/04/2020 & sử dụng công nghệ (UTAUT) và kết hợp thêm yếu tố rủi ro. \\
\hline Ngày nhận lại: 09/08/2020 & Nghiên cứu này khảo sát 155 nông dân và 20 thương lái tại tỉnh \\
\hline Duyệt đăng: 18/08/2020 & $\begin{array}{l}\text { Tiền Giang. Kết quả nghiên cứu cho thấy các yếu tố kỳ vọng hiệu } \\
\text { quả, dễ sử dụng, thái độ hướng tới sử dụng công nghệ, và ảnh } \\
\text { hưởng xã hội có quan hệ cùng chiều với việc sử dụng Ethephon. } \\
\text { Hơn nữa, kết quả còn cho thấy sự lo lắng và rủi ro có quan hệ } \\
\text { ngược chiều với việc sử dung Ethephon cho trái sầu riêng. }\end{array}$ \\
\hline
\end{tabular}

Tù khóa:

Ethephon; hành vi sử dụng; sầu riêng sau thu hoạch; Tiền Giang

ABSTRACT
This paper investigates some determinants of the intention to
use the Ethephon for ripening of post-harvest Durian. The
research model was developed based on the Unified Theory of
Acceptance and Use of Technology (UTAUT) and combined with
a perceived risk. This study surveyed 155 farmers and 20
merchants in Tien Giang Province. The results show that
performance expectancy, perceived ease of use, attitude toward
using technology, and social influence have a significant positive
influence on the usage of the Ethephon. In addition, results also
show that anxiety and perceived risk have a significant negative
effect on using the Ethephon for post-harvest durian.

Keywords:

Ethephon; behavioral use; postharvest Durian; Tien Giang

effect on using the Ethephon for post-harvest durian.

\section{Giới thiệu}

Sầu riêng có tên khoa học là Durio zibethinus. Sầu riêng có hương vị đặc biệt, rất giàu dinh dưỡng và được nhiều người ưa thích. Tại Việt Nam, sầu riêng được trồng tập trung tại một số tỉnh Đồng Bằng Sông Cửu Long (ĐBSCL) như Tiền Giang, Bến Tre, Vĩnh Long, Cần Thơ và một số tỉnh Đông Nam Bộ và Tây nguyên như Bình Dương, Đồng Nai, Bình Phước, và Lâm Đồng. Riêng tại ĐBSCL, sầu riêng được trồng nhiều nhất ở tỉnh Tiền Giang (Vu \& Vo, 2011). Hiện nay, sầu riêng là một trong những cây trồng quan trọng tại Tiền Giang. Diện tích trồng sầu riêng ở Tiền Giang hiện nay khoảng 13,000 ha, năng suất khoảng 22 tấn/ha. Với giá 
sầu riêng dao động từ 40 nghìn/kg đến 90 nghìn/kg, thu nhập từ sầu riêng trên 10 nghìn tỉ đồng và trở thành cây ăn trái đặc sản của tỉnh Tiền Giang. Sau khi trừ chi phí, nông dân đạt lãi ròng khoảng 1 tỷ/ha. Sầu riêng đã góp phần nâng cao thu nhập và cải thiện đời sống của người nông dân trồng sầu riêng.

Khi canh tác sầu riêng với qui mô lớn, nông dân không thể chờ rụng từng trái chín. Hơn nữa, trong xuất khẩu sẽ cần thời gian vận chuyển, nếu trái chín rụng tự nhiên khi đến nơi tiêu thụ sẽ quá chín và giảm chất lượng. Thông thường nông dân sẽ thu hoạch đồng loạt khi trái sắp chín, sau đó sử dụng chất chín trái để giúp trái chín đồng loạt và kiểm soát thời gian trái chín. Nhiều nhà khoa học cho rằng việc sử dụng chất chín trái, tên thương mại là Ethephon, được xem là ứng dụng công nghệ vào quá trình sau thu hoạch và được sử dụng tại nhiều quốc gia như Mỹ, Canada, Italy, Chile, Australia (Duong \& Nguyen, 2018; Le, Le, \& Pham, 2012; Phuong Hoa, 2016; Thuc Vy, 2015). Năm 2015, tại tọa đàm khoa học về Ethephon do Hiệp hội doanh nghiệp nông nghiệp và trang trại Việt Nam phối hợp với Hội doanh nghiệp hàng Việt Nam chất lượng cao tổ chức tại Thành phố Hồ Chí Minh, nhiều chuyên gia cũng cho rằng việc sử dụng Ethephon không độc và có thể ứng dụng trong sau thu hoạch (Thuc Vy, 2015). Tại nước ta, Ethephon chưa được đăng ký sử dụng trong bảo quản nông sản nhưng đã được sử dụng khá phổ biến trong hỗ trợ chín trái sầu riêng. Hiện tại, tất cả những loại thuốc thúc chín trái trên thị trường chưa được Bộ Nông nghiệp và Phát triển Nông thôn cấp phép, điều này làm cho người tiêu dùng không an tâm. Các loại thuốc thúc chín trái vẫn chưa có trong danh mục thuốc được phép sử dụng nên có thể nói việc sử dụng Ethephon là bất hợp pháp (Xuan Ngoc, 2014). Lợi dụng việc sử dụng Ethephon khá phổ biến và chưa được kiểm soát, một số đơn vị đã sử dụng thêm một số chế phẩm hóa học không rõ nguồn gốc và một số chất có thể gây độc hoặc thúc trái chín sớm. Đây là mối lo ngại rất lớn của người tiêu dùng Việt Nam. Các cơ quan chức năng đã xử phạt một số cơ sở thu mua sầu riêng nhúng thuốc thúc chín trái (Hoai Thanh, 2016; Thanh Bac \& Hoai Thuong, 2013; Trung Tan, 2015).

Theo các chuyên gia, việc sử dụng Ethephon không hại cho người tiêu dùng và nên khuyến khích sử dụng nếu được kiểm soát tốt để giúp cho quá trình thu hoạch trái sầu riêng thuận lợi, góp phần hiện đại hóa nền nông nghiệp. Xét trên khía cạnh đó, việc sử dụng Ethephon có thể xem là áp dụng khoa học công nghệ, cụ thể là công nghệ sau thu hoạch. Vì vậy, chúng ta có thể sử dụng lý thuyết hợp nhất về chấp nhận và sử dụng công nghệ (UTAUT: Unified Theory of Acceptance and Use of Technology) được đề xuất bởi Venkatesh, Morris, Davis, và Davis (2003) để giải thích việc sử dụng Ethephon (Venkatesh et al., 2003). Đây là mô hình đã được ứng dụng rộng rãi để xem xét các yếu tố ảnh hưởng đến việc sử dụng công nghệ và đang được nghiên cứu bổ sung cho phù hợp hơn ở hiện tại (Dwivedi, Rana, Jeyaraj, Clement, \& Williams, 2019). Các mô hình chấp nhận công nghệ có thể sử dụng để đánh giá việc chấp nhận của nông dân liên quan đến việc áp dụng công nghệ trong nông nghiệp (Andualem et al., 2018; Flett et al., 2004; Folorunso \& Ogunseye, 2008; Obiero et al., 2019). Ở Việt Nam, đã có nghiên cứu về mô hình UTAUT trong việc không sử dụng chất cấm trong nuôi heo (T. T. T. Nguyen \& Nguyen, 2017) nhưng chúng tôi chưa tìm thấy nghiên cứu về UTAUT cho việc sử dụng Ethephon cho trái sầu riêng.

Mục tiêu của nghiên cứu này là xác định những yếu tố ảnh hưởng đến việc sử dụng Ethephon cho trái sầu riêng sau thu hoạch chủ yếu dựa trên mô hình UTAUT. Trên cơ sở đó, bài báo sẽ đề xuất một số gợi ý để đảm bảo việc sử dụng Ethephon hiệu quả, an toàn. Sau phần giới thiệu phần còn lại của bài báo được tổ chức như sau: phần 2 là cơ sở lý thuyết và giả 
thuyết nghiên cứu, phần 3 là phương pháp nghiên cứu và thang đo, phần 4 là kết quả và thảo luận, và cuối cùng là kết luận.

\section{Cơ sở lý thuyết và giả thuyết nghiên cứu}

\subsection{Chế phẩm hóa học Ethephon}

Chế phẩm hóa học là sản phẩm được tạo ra từ những chất hóa học. Ethephon có tên khoa học là 2-chloroethylphosphonic acid $\left(\mathrm{C}_{2} \mathrm{H}_{6} \mathrm{ClO}_{3} \mathrm{P}\right)$, được phát hiện vào năm 1965 và đăng ký sử dụng đầu tiên tại Mỹ vào năm 1973. Ethephon là chất điều hoà sinh trưởng thực vật có tác dụng kích thích trái chín. Ethephon có nhiều tên thương mại khác nhau, tại Mỹ là Ethrel, tại Đức là Flodimex, và tại Nga là Ethephon. Thử nghiệm cho thấy Ethephon không độc (Tran, 2020).

\subsection{Lý thuyết hợp nhất về chấp nhận và sử dụng công nghệ}

Mô hình chấp nhận công nghệ (TAM) được đề xuất đầu tiên bởi Davis, Bagozzi, và Warshaw (1989) để xem xét các yếu tố ảnh hưởng đến việc chấp nhận và sử dụng sản phẩm công nghệ thông tin gồm các yếu tố: kỳ vọng hiệu quả, dễ áp dụng, và thái độ hướng tới sử dụng công nghệ (Davis et al., 1989). Sau đó, mô hình này được mở rộng và kết hợp nhiều mô hình khác để tạo thành lý thuyết hợp nhất về chấp nhận và sử dụng công nghệ (UTAUT: Unified Theory of Acceptance and Use of Technology). Mô hình này được đề xuất bởi Venkatesh và cộng sự (2003) và bổ sung 2 yếu tố là điều kiện thuận lợi và ảnh hưởng xã hội so với mô hình TAM (Venkatesh et al., 2003).

Các mô hình chấp nhận công nghệ có thể sử dụng để xem xét các yếu tố ảnh hưởng đến việc chấp nhận công nghệ của nông dân. Mô hình TAM được nghiên cứu để giải thích thỏa đáng việc áp dụng và sử dụng các công nghệ trong chăn nuôi bò sữa (Flett et al., 2004). Trong nghiên cứu về việc chấp nhận công nghệ trong triển khai các phần mềm hỗ trợ cho nông nghiệp, các yếu tố trong mô mình TAM là tương đối phù hợp (Folorunso \& Ogunseye, 2008). Nghiên cứu sự chấp nhận sử dụng tin nhắn di động để cung cấp thông tin liên quan đến trang trại của nông dân Ý đã dựa trên lý thuyết hợp nhất về chấp nhận và sử dụng công nghệ mở rộng (UTAUT2) (Andualem et al., 2018). Mô hình ứng dụng công nghệ cũng được nghiên cứu trong nuôi trồng thủy sản ở Châu Phi (Obiero et al., 2019).

\subsection{Kỳ vọng hiệu quả}

Kỳ vọng hiệu quả là mức độ mà một cá nhân tin rằng việc sử dụng hệ thống sẽ giúp họ có thể tăng hiệu suất công việc (Davis, 1989). Trong mô UTAUT cũng như các nghiên cứu về ứng dụng của mô hình này trong nông nghiệp, kỳ vọng hiệu quả có quan hệ cùng chiều với việc sử dụng công nghệ (Andualem et al., 2018; Flett et al., 2004; Folorunso \& Ogunseye, 2008; Venkatesh et al., 2003). Việc sử dụng Ethephon được kỳ vọng sẽ mang lại hiệu quả trong việc giúp trái sầu riêng chín ngon hơn (Thuc $\mathrm{Vy}, 2015)$. Qua thời gian sử dụng thực tế đã mang lại những hiệu quả tích cực trong việc hỗ trợ nông dân thu hoạch sầu riêng thuận lợi, và đảm bảo chất lượng. Như vậy, giả thuyết thứ nhất được đề xuất như sau:

H1: Kỳ vọng hiệu quả có quan hệ cùng chiều với việc sủ dụng Ethephon cho trái sầu riêng sau thu hoạch

\subsection{Dễáp dụng}

Dễ áp dụng là mức độ dễ dàng kết hợp với các yếu tố khác trong việc sử dụng hệ thống. Tương tự như yếu tố kỳ vọng hiệu quả, theo UTAUT và nghiên cứu về ứng dụng công nghệ trong nông nghiệp, yếu tố này có quan hệ cùng chiều với việc sử dụng công nghệ (Andualem et al., 
2018; Flett et al., 2004; Folorunso \& Ogunseye, 2008; Venkatesh et al., 2003). Dễ áp dụng trong nghiên cứu này được hiểu là một cá nhân tin rằng việc sử dụng Ethephon sẽ không tốn nhiều công sức. Người sử dụng Ethephon chỉ cần pha với nước, rồi nhúng trái sầu riêng vào. Hơn nữa, Ethephon không gây độc, không có mùi nên làm việc với chất này khá thuận tiện mà không cần dụng cụ bảo hộ đặc biệt. Các thao tác trong sử dụng Ethephon cho trái sầu siêng là khá dễ dàng và dự báo có quan hệ cùng chiều với việc sử dụng. Do đó, giả thuyết tiếp theo như sau:

H2: Dễ áp dụng tác động cùng chiều với việc sủ dụng Ethephon cho trái sầu riêng sau thu hoạch

\subsection{Thái độ hướng đến sử dụng công nghệ}

Thái độ là cảm xúc tích cực hay tiêu cực của một cá nhân về việc thực hiện các hành vi mục tiêu. Nghiên cứu ứng dụng công nghệ trong nông nghiệp cho thấy yếu tố này có ảnh hưởng đến việc sử dụng công nghệ (Obiero et al., 2019). Nông dân và thương lái có thể cảm thấy phấn khởi trong việc sử dụng Ethephon vì đã giúp cho việc thu hoạch được thuận lợi, giúp quá trình xuất khẩu dễ dàng, và đảm bảo chất lượng trái sầu riêng. Phương pháp chờ trái rụng chín trước kia không thể áp dụng cho canh tác quy mô lớn. Chúng ta có thể dự báo rằng người sử dụng Ethephon có thái độ tích cực đối với việc sử dụng chất chín trái nên đề xuất giả thuyết thứ ba như sau:

H3: Thái độ hương tới sủ dụng công nghệ có tác cùng chiều đến việc sủ dụng Ethephon cho trái sầu riêng sau thu hoạch

\section{6. Änh hưởng xã hội}

Ảnh hưởng xã hội là mức độ ảnh hưởng mà một cá nhân nhận thức rằng những người quan trọng khuyên nên sử dụng công nghệ. Nghiên cứu trước đây chỉ ra rằng, yếu tố này có ảnh hưởng đáng kể đến quyết định hành vi của người tiêu dùng (Venkatesh et al., 2003). Đối với việc canh tác sầu riêng, người dân có thể bị ảnh hưởng bởi người nông dân xung quanh, thương lái, khách hàng, và người bán chất chín trái. Do đó, giả thuyết thứ tư được đề xuất như sau:

H4: Anh hưởng xã hội ảnh hương tích cực đến việc sủ dụng Ethephon cho trái sầu riêng sau thu hoạch

\section{7. Điều kiện thuận Iọi}

Các điều kiện thuận lợi là mức độ mà một cá nhân tin rằng cơ sở hạ tầng kỹ thuật được tổ chức để hỗ trợ sử dụng công nghệ. Điều kiện thuận lợi có ảnh hưởng đến quyết định sử dụng công nghệ (Venkatesh et al., 2003). Trong việc sử dụng Ethephon cho trái sầu riêng, điều kiện thuận lợi bao gồm các khía cạnh liên quan giúp việc sử dụng Ethephon thuận lợi và dự đoán có quan hệ cùng chiều với việc sử dụng Ethephon. Giả thuyết thứ năm như sau:

H5: Điều kiện thuận lợi có tác cùng chiều đến việc sủ dụng Ethephon cho trái sầu riêng sau thu hoach

\subsection{Sụ̂ lo lắng}

Sự lo lắng được định nghĩa là tâm lý lo âu hay cảm xúc phản ứng khi thực hiện một hành vi cụ thể. Lo lắng liên quan đến sự không chắc chắn và những hậu quả liên quan đến hành động của người sử dụng. Sự lo lắng có thể tác động tiêu cực đến quyết định hành vi của người sử dụng (Compeau, Higgins, \& Huff, 1999). Trong canh tác sầu riêng, sự lo lắng có thể xuất phát từ việc sử dụng không đúng cách, không hiệu quả, bị tố giác, hoặc bị phạt như một số trường hợp báo chí đã nêu. Như vậy, giả thuyết thứ sáu được đề xuất như sau: 
thu hoach

H6: Sự lo lắng có quan hệ ngược chiều đến việc sử dụng Ethephon cho trái sầu riêng sau

\subsection{Cảm nhận rủi ro}

Rủi ro được xem là sự không may mắn, hoặc sự tổn thất. Ở nước ta hiện nay, Ethephon chưa chính thức được phép sử dụng cho trái sầu riêng nên tiềm ẩn nhiều rủi ro. Nhiều đơn vị từng sử dụng cũng đã bị phạt (Hoai Thanh, 2016). Các sản phẩm bán trên thị trường được gắn với tên phân bón lá nên việc sử dụng chất chín trái có một số rủi ro nhất định. Trong nghiên cứu này, rủi ro là những bất trắc ngoài ý muốn xảy ra trong quá trình sử dụng Ethephon. Bên cạnh các yếu tố trong UTAUT, nghiên cứu này xem xét thêm yếu tố rủi ro trong việc sử dụng Ethephon. Yếu tố này cũng được xem xét trong các nghiên cứu liên quan đến ý định sử dụng và hành vi sử dụng công nghệ (Martins, Oliveira, \& Popovič, 2014; P. D. Nguyen, Nguyen, \& Angelina, 2018). Rủi ro có thể tác động ngược chiều với việc sử dụng Ethephon nên giả thuyết cuối cùng đề xuất như sau: thu hoach

H7: Rủi ro có tác động ngược chiều với việc sủ dụng Ethephon cho trái sầu riêng sau

\section{Thiết kế nghiên cứu và thang đo}

\subsection{Thiết kế nghiên cứu}

Đầu tiên, bài báo xây dựng thang đo nháp từ việc tổng hợp ở các nghiên cứu liên quan đến mô hình UTAUT. Sau đó, chúng tôi đã tiến hành phỏng vấn gồm 16 nông dân và 04 thương lái bằng phương pháp phỏng vấn tay đôi. Mục đích của phỏng vấn cần giải quyết ba vấn đề chính gồm: kiểm tra những yếu tố ảnh hưởng đến việc sử dụng Ethephon cho trái sầu riêng sau thu hoạch, điều chỉnh và bổ sung các câu hỏi khảo sát, và kiểm tra từ ngữ trong từng câu hỏi nhằm đảm bảo phần đông đối tượng khảo sát hiểu đúng. Nghiên cứu này sử dụng thang đo khá phổ biến trong các nghiên cứu trước là thang đo Likert.

Tiếp theo, nghiên cứu này sử dụng phương pháp định lượng thông qua khảo sát chính thức. Việc thu thập thông tin được tiến hành bằng cách phát phiếu khảo sát trực tiếp. Dữ liệu sau khảo sát sẽ được kiểm tra và làm sạch dữ liệu. Tổng số bảng khảo sát thu về là 175 phiếu, do khảo sát trực tiếp nên thông tin trong các câu trả lời là đầy đủ và có độ tin cậy cao. Số lượng mẫu này đảm bảo điều kiện cho phân tích nhân tố và hồi quy (T. D.Nguyen, 2011, pp. 398-499).

Cuối cùng, nghiên cứu này sử dụng phần mềm SPSS để xử lý dữ liệu. Bước đầu tiên là đánh giá độ tin cậy của thang đo thông qua hệ số Cronbach Alpha. Thứ hai là phân tích nhân tố để xác định các yếu tố ảnh hưởng đến việc sử dụng Ethephon. Bước cuối cùng là sử dụng hồi quy để kiểm định giả thuyết và nhận xét kết quả nghiên cứu.

\subsection{Thang đo}

Kỳ vọng hiệu quả được đo lường thông qua sáu câu hỏi gồm ba câu hỏi dựa trên Venkatesk và cộng sự (2003) gồm: tôi nhận thấy chất này hữu ích trong xử lý chín trái (HQ1), việc sử dụng chất này cho phép chín trái nhanh hơn (HQ2), việc sử dụng chất này giúp tăng năng suất (HQ3), và ba câu hỏi dựa vào nghiên cứu định tính gồm: việc sử dụng chất này giúp trái chín ngon hơn (HQ4), việc sử dụng chất này hạn chế suy cây sầu riêng (HQ5), và việc sử dụng chất này không độc hại cho người tiêu dùng (HQ6). 
Yếu tố dễ sử dụng được đo lường thông qua hai câu hỏi dựa trên mô hình UTAUT gồm: học cách sử dụng chất này dễ dàng $(\mathrm{AD} 1)$, tôi nhận thấy chất này dễ sử dụng (AD3) (Venkatesh et al., 2003) và ba câu hỏi dựa vào nghiên cứu định tính gồm: thao tác sử dụng chất này dễ dàng $(\mathrm{AD} 2)$, sử dụng chất này không mất thời gian $(\mathrm{AD} 4)$, và giá thành chất chín trái rẻ $(\mathrm{AD} 5)$.

Đo lường thái độ hướng tới sử dụng công nghệ hoàn toàn dựa vào mô hình UTAUT gồm: việc sử dụng chất chín trái là một ý tưởng hay (TD1), tôi phấn khởi khi sử dụng chất chín trái (TD2), tôi thích sử dụng chất chín trái (TD3), tôi cảm thấy vui vẻ khi sử dụng chất chín trái (TD4) (Venkatesh et al., 2003).

Ảnh hưởng xã hội đo lường thông qua một câu hỏi từ nghiên cứu định tính: có người xung quanh sử dụng $(\mathrm{XH} 1)$, và ba câu hỏi dựa vào nghiên cứu của $\mathrm{T}$. T. T. Nguyen và Nguyen (2017) gồm: theo yêu cầu của thương lái (XH2), người tiêu dùng thích trái chín ngon (XH3), và được sự hướng dẫn của người bán chất chín trái (XH4).

Điều kiện thuận lợi gồm ba câu hỏi: tôi có kiến thức để sử dụng (TL1) (Venkatesh et al., 2003), việc sử dụng không phiền phức (TL2) dựa vào nghiên cứu định tính, và luôn có sẵn trên thị trường (dễ mua) (TL3) (T. T. T. Nguyen \& Nguyen, 2017).

Sự lo lắng được đo lường thông qua nghiên cứu định tính gồm: tôi lo ngại sẽ sử dụng không đúng cách (LL1), tôi lo ngại sử dụng không đạt hiệu quả (LL2) và nghiên cứu của $T$. T. T. Nguyen và Nguyen (2017) gồm: tôi lo ngại người tiêu dùng không mua (LL3), tôi lo ngại người khác tố giác (LL4), và tôi lo ngại bị phạt (tiền, tù, hủy sầu riêng) (LL5).

Yếu tố rủi ro được đo lường thông qua các câu hỏi dựa trên nghiên cứu của $T$. T. T. Nguyen và Nguyen (2017) gồm: kiểm soát của nhà nước (RR1), chất chín trái không đảm bảo chất lượng (RR2), không rõ nguồn gốc chất chín trái (RR3), và người bán không đảm bảo hiệu quả chất chín trái (RR4).

Trong mô hình UTAUT2, tác giả đo lường việc sử dụng công nghệ thông qua tần suất sử dụng công nghệ (Venkatesh, Thong, \& Xu, 2012). Trong nghiên cứu về mua vé máy bay trực tuyến, nhóm tác giả đo lường thông qua tần suất sử dụng các trang web thương mại điện tử của công ty hàng không để mua vé (Rodríguez \& Trujillo, 2013). Tương tự, nghiên cứu này sử dụng câu hỏi như sau: sầu riêng của tôi thường sử dụng chất chín trái (SD).

Các nghiên cứu trước sử dụng thang đo Likert, chủ yếu là thang đo 7 mức (Dwivedi et al., 2019; Venkatesh et al., 2012), hoặc 5 mức (Andualem et al., 2018; T. T. T. Nguyen \& Nguyen, 2017). Ở đây, đề tài chọn thang đo Likert 5 mức.

\section{Thảo luận kết quả}

\subsection{Mô tả mẫu}

Nghiên cứu này chọn mẫu theo phương pháp lấy mẫu ngẫu nhiên và thu về 175 phiếu khảo sát. Đối tượng khảo sát là những người trực tiếp tham gia canh tác sầu riêng nên giới tính nam chiếm $65.7 \%$, và nông dân chiếm $88.6 \%$. Đối tượng khảo sát khá đa dạng về tuổi tác, tuổi dưới 35 tuổi chiếm $31.4 \%$, tuổi từ 35 đến 45 chiếm $37.2 \%$, tuổi trên 45 chiếm $31.4 \%$. Học vấn của nông dân và thương lái chủ yếu từ trung học phổ thông trở xuống, trình độ trên trung học phổ thông chiếm $8.6 \%$. Tiểu học chiếm $22.9 \%$, trung học cơ sở chiếm $37.1 \%$, trung học phổ thông chiếm 31.4\%. Thâm niên của đối tượng khảo sát chủ yếu từ 05 đến 10 năm chiếm $45.7 \%$, dưới 05 năm là $28.6 \%$, và trên 10 năm là $25.7 \%$. 


\subsection{Kiểm định độ tin cậy}

Đối với nhân tố kỳ vọng hiệu quả, có ba biến (câu hỏi) bị loại do có hệ số tương quan biến - tổng không đạt yêu cầu $(<0.30)$ gồm giúp tăng năng suất (HQ3), hạn chế suy cây sầu riêng (HQ5), và không độc hại cho người tiêu dùng (HQ6). Vậy, kỳ vọng hiệu quả trong việc sử dụng Ethephon chỉ còn ba câu hỏi và hệ số Cronbach alpha cho thang đo này là 0.82 và hệ số tương quan biến - tổng thấp nhất là 0.51 . Đối với nhân tố dễ áp dụng, hệ số Cronbach alpha của thang đo này là 0.85 , hệ số tương quan biến - tổng thấp nhất là 0.61 , và chấp nhận tất cả năm câu hỏi. Hệ số Cronbach Alpha của thang đo thái độ hướng tới sử dụng công nghệ là 0.83 , hệ số tương quan biến - tổng thấp nhất là 0.64 , và loại câu hỏi việc sử dụng chất chín trái là một ý tưởng hay (TD1). Đối với nhân tố ảnh hưởng xã hội, câu hỏi theo yêu cầu của thương lái (XH2) có hệ số tương quan biến - tổng là 0.25 nên bị loại. Hệ số Cronbach Alpha của yếu tố ảnh hưởng xã hội sau khi loại biến là 0.80 và hệ số tương quan biến - tổng thấp nhất là 0.48 . Hệ số Cronbach Alpha cho thang đo điều kiện thuận lợi là 0.84 và hệ số tương quan biến - tổng thấp nhất là 0.55 nên thang đo này đạt yêu cầu. Đối với nhân tố sự lo lắng, yếu tố tôi lo ngại bị phạt (tiền, tù, hủy sầu riêng) (LL5) có hệ số tương quan biến - tổng là 0.22 . Sau khi loại biến này, Cronbach alpha cho thang đo này là 0.76 và hệ số tương quan biến - tổng thấp nhất là 0.344 . Đối với nhân tố rủi ro, câu hỏi kiểm soát của nhà nước (RR1) gần như không có tương quan với rủi ro, khi hệ số tương quan biến - tổng là -0.14 . Hệ số Cronbach alpha sau khi loại biến này là 0.78 và hệ số tương quan biến - tổng thấp nhất là 0.58 .

\subsection{Phân tích nhân tố}

Ở đây, nghiên cứu sử dụng phương pháp trích PAF (Principal Axis Factoring) và phép quay Promax. Sau khi loại các biến HQ3, HQ5, HQ6, TD1, XH2, LL5, RR1 ở bước đánh giá độ tin cậy thang đo, mô hình còn lại 24 biến. Kết quả phân tích EFA cho thấy có ba biến quan sát $\mathrm{AD} 4, \mathrm{AD} 5, \mathrm{LL} 4$ có hệ số factor loading $<0.5$ nên loại khỏi phân tích. Sau khi loại biến AD4 và $\mathrm{AD}$, hệ số Cronbach Alpha của yếu tố dễ áp dụng là 0.81 và hệ số tương quan biến - tổng thấp nhất là 0.59 . Đối với biến LL4, kết quả này phù hợp với bước đánh giá độ tin cậy thang đo khi hệ số tương quan biến - tổng khá thấp (0.34).

Sau khi loại ba biến trên, kết quả phân tích nhân tố có $\mathrm{KMO}=0.569>0.5$ nên phân tích nhân tố có thể chấp nhận được và Sig. (Barlett's Test) $=0.000<0.05$ chứng tỏ các biến quan sát có tương quan với nhau trong tổng thể (T. D. Nguyen, 2011, pp. 396-397). Kết quả phân tích cho thấy nhân tố thứ 07 có hệ số Eigenvalues $=1.171>1$ nên tất cả các biến đo lường trích được 07 nhân tố. Tổng phương sai trích (Rotation Sums of Squared Loadings (Cumulative \%)) là $71.257 \%>50 \%$. Điều này chứng tỏ $71.257 \%$ biến thiên của dữ liệu được giải thích bởi 07 nhân tố, và 21 biến quan sát gom thành 07 nhân tố.

\subsection{Kiểm định giả thuyết}

Kết quả kiểm định giả thuyết thông qua hồi quy như Bảng 1 . Xét mức ý nghĩa $5 \%$, các yếu tố "kỳ vọng hiệu quả", "dễ áp dụng", "thái độ hướng tới sử dụng công nghệ", và "ảnh hưởng xã hội” có mối quan hệ cùng chiều với "việc sử dụng Ethephon cho trái sầu riêng sau thu hoạch." Nhìn lại kiểm định đa cộng tuyến, nhận thấy $\mathrm{VIF}<2$, xét một cách tổng quát là đạt yêu cầu. Dựa vào hệ số beta chuẩn hóa, chúng ta thấy "thái độ hướng tới sử dụng công nghệ" có tác động mạnh nhất đối với việc sử dụng Ethephon. Trong khi đó, các yếu tố "sự lo lắng", và "rủi ro" có mối quan hệ ngược chiều với "việc sử dụng Ethephon cho trái sầu riêng sau thu hoạch." Như vậy, chúng ta chấp nhận các giả thuyết $\mathrm{H1}$, H2, H3, H4, H6, và H7. 


\section{Bảng 1}

Kết quả hồi quy

\begin{tabular}{|c|c|c|c|c|c|c|}
\hline \multirow{2}{*}{ Mô hình } & \multicolumn{2}{|c|}{ Hệ số chưa chuẩn hóa } & Hệ số chuẩn hóa & \multirow{2}{*}{ Sig. } & \multicolumn{2}{c|}{ Đa cộng tuyến } \\
\cline { 2 - 4 } & B & Std. Error & Beta & & Dung sai & VIF \\
\hline (Hằng số) & 1.000 & .515 & & .054 & & \\
\hline HQ & .373 & .078 & .199 & .000 & .927 & 1.078 \\
\hline AD & .257 & .058 & .206 & .000 & .727 & 1.375 \\
\hline TD & .353 & .046 & .350 & .000 & .776 & 1.289 \\
\hline XH & .213 & .073 & .149 & .004 & .617 & 1.620 \\
\hline TL & .033 & .064 & .024 & .606 & .732 & 1.366 \\
\hline LL & -.353 & .046 & -.334 & .000 & .838 & 1.193 \\
\hline RR & -.227 & .040 & -.242 & .000 & .902 & 1.108 \\
\hline
\end{tabular}

Nguồn: Kết quả phân tích số liệu (2020)

“Điều kiện thuận lợi” không có quan hệ thống kê đối với “việc sử dụng Ethephon cho trái sầu riên sau thu hoạch" do sig =0.606 > 0.01. Như vậy, chúng ta bác bỏ giả thuyết H5.

\subsection{Thảo luận}

Các cơ quan có trách nhiệm cần khẳng định tính hợp pháp của việc sử dụng Ethephon. Do chất này chưa chính thức được phép sử dụng nên đã tạo ra rào cản làm hạn chế việc sử dụng chất chín trái. Dựa vào kết quả hồi qui, chúng ta thấy yếu tố sự lo lắng, và rủi ro có ảnh hưởng mạnh đến việc hạn chế sử dụng Ethephon. Để làm được điều này, các cơ quan chức năng cần tổ chức hội thảo có sự tham gia các nhà khoa học, các chuyên gia trong lĩnh vực công nghệ sau thu hoạch, các chuyên gia trong lĩnh vực chế biến và bảo quản nông sản, tham khảo việc sử dụng Ethephon ở các nước để có những đánh giá đầy đủ, khách quan nhằm mang lại những lợi ích chính đáng cho người nông dân canh tác sầu riêng nhưng vẫn đảm bảo an toàn cho người tiêu dùng trong và ngoài nước.

Xét trường hợp Ethephon không độc và thực tế đã được sử dụng khá phổ biến trong thời gian qua thì chúng ta cần có các giải pháp xóa bỏ những rào cản trong việc sử dụng Ethephon gồm giảm sự lo lắng, và rủi ro của nông dân và thương lái.

Thứ nhất, các cơ quan chức năng cần công khai việc cho phép sử dụng, ban hành văn bản hướng dẫn sử dụng Ethephon, và có cơ chế kiểm soát phù hợp để mang lại lợi ích kinh tế chính đáng cho trái sầu riêng cũng như là tạo sự an tâm cho người tiêu dùng. Các cơ quan cũng nên tổ chức tập huấn hướng dẫn nông dân, thương lái quy trình sử dụng Ethephon. Đồng thời, cơ quan chức năng cần tăng cường kiểm tra, giám sát, phạt nặng các đơn vị bán các chất không đảm bảo chất lượng và không rõ nguồn gốc, và yêu cầu họ phải cam kết bán Ethephon có chất lượng. Các cơ quan ngôn luận và các phương tiện truyền thông cần hỗ trợ cơ quan nhà nước để cung cấp thông tin có sơ sở khoa học, khách quan tránh gây hiểu lầm, làm thiệt hại đến lợi ích chính đáng của người nông dân trồng sầu riêng. Đẩy mạnh tuyên truyền giúp người tiêu dùng an tâm sử dụng sầu riêng. 
Thứ hai, nông dân và thương lái cần tham dự đầy đủ các khóa tập huấn để hiểu và tuân thủ nghiêm quy trình sử dụng Ethephon sao cho đảm bảo chất lượng của sầu riêng, và an toàn đối với người tiêu dùng. Người nông dân cần phải để trái sầu riêng đủ già, và sử dụng đúng liều lượng như quy định. Hơn nữa, chúng ta cần có giải pháp truy xuất nguồn gốc, sổ ghi chép hoặc số hóa thông tin về quá trình canh tác sầu riêng từ lúc chăm sóc cho đến người tiêu dùng nhằm kiểm soát việc sử dụng Ethephon đúng giai đoạn và liều lượng phù hợp. Đồng thời, có thể phát triển các thiết bị kiểm tra tồn dư Ethephon để thực hiện hậu kiểm trước khi bán trái sầu riêng cho người tiêu dùng. Khi chính thức được phép sử dụng, nông dân và thương lái sẽ hoàn toàn an tâm, và không lo lắng khi sử dụng chất này. Khi đó, nông dân và thương lái sẽ có thái độ tích cực trong việc sử dụng Ethephon. Các yếu tố về lo ngại bị tố giác, bị phạt, kiểm soát của nhà nước sẽ bị triệt tiêu. Nông dân và thương lái không còn phải sử dụng Ethephon được bán ra thị trường với tên phân bón lá và chưa có hướng dẫn chính thức từ các nhà sản xuất. Thay vào đó, nông dân và thương lái sẽ được sử dụng Ethephon có nguồn gốc rõ ràng, và đảm bảo hiệu quả từ nhà cung cấp.

Thứ ba, người tiêu dùng cũng cần có những quyết định thông minh khi nền nông nghiệp chúng ta đang trong quá trình công nghiệp hóa, chúng ta không thể canh tác theo tự nhiên như trước kia thì việc sử dụng các công nghệ, trong đó có công nghệ sau thu hoạch cũng là việc hoàn toàn bình thường.

Xét trường hợp ngược lại, nếu chất này có thể gây độc cho người tiêu dùng thì chúng ta cần sử dụng các biện pháp để nhằm hạn chế việc sử dụng Ethephon cho sầu riêng như tuyên truyền cho người nông dân và thương lái hiểu được các tác hại khi sử dụng Ethephon. Việc sử dụng Ethephon có thể dẫn đến người tiêu dùng tẩy chai trái sầu riêng, và làm giảm giá sầu riêng, gây ảnh hưởng đến hiệu quả kinh tế trái sầu riêng. Đồng thời, cơ quan chức năng cần tăng mức phạt đối với những người mua bán và sử dụng Ethephon cho trái sầu riêng sau thu hoạch.

\section{Kết luận}

Đề tài đã khảo sát 175 nông dân và thương lái về việc sử dụng Ethephon cho trái sầu riêng thu hoạch chủ yếu dựa trên lý thuyết hợp nhất về chấp nhận và sử dụng công nghệ. Kết quả phân tích dữ liệu cho thấy, kỳ vọng hiệu quả, dễ áp dụng, thái độ hướng đến sử dụng công nghệ, và ảnh hưởng xã hội có quan hệ cùng chiều với việc sử dụng Ethephon. Trong khi, các yếu tố sự lo lắng và rủi ro có quan hệ ngược chiều với việc sử dụng Ethephon cho trái sầu riêng. Các cơ quan chức năng có liên quan cần xem xét, đánh giá và quyết định tính hợp pháp của việc sử dụng Ethephon cho trái sầu riêng. Nếu chất này có thể sử dụng thì cần xóa bỏ những rào cản giúp quá trình thu hoạch trái sầu riêng được tiện lợi cũng như giúp trái sầu riêng chín ngon hơn. Mô hình nghiên cứu này có thể mở rộng trong việc xác định những yếu tố thúc đẩy cũng như cản trở trong việc sử dụng công nghệ trong nông nghiệp. Từ đó, chúng ta có những giải pháp phù hợp hơn trong quá trình hiện đại nền nông nghiệp nước nhà theo xu thế của các nước phát triển.

\section{Tài liệu tham khảo}

Andualem, E., Reidsma, P., Poortvliet, M., Belay, M., Bijen, B., \& Kooistra, L. (2018). Exploring farmers' intentions to adopt mobile Short Message Service (SMS) for citizen science in agriculture. Computers and Electronics in Agriculture, 151(2018), 295-310. doi:10.1016/j.compag.2018.06.015

Compeau, D., Higgins, C., \& Huff, S. (1999). Social cognitive theory and individual reactions to computing technology: A longitudinal study. MIS Quarterly, 23(2), 145-158. doi: $10.2307 / 249749$ 
Davis, F. D. (1989). Perceived usefulness, perceived ease of use, and user acceptance of information technology. MIS Quarterly, 13(3), 319-340. doi:10.2307/249008

Davis, F. D., Bagozzi, R. P., \& Warshaw, P. R. (1989). User acceptance of computer technology: A comparison of two theoretical models. Management Science, 35(8), 982-1003.

Duong, N. T. C., \& Nguyen, P. V. (2018). Nghiên cứu quá trình rấm chín quả sầu riêng Ri 6 bằng tác động của khí ethylen ngoại sinh [Study on the ripening process of Ri 6 durian by the effect of exogenous ethylene gas]. Khoa học Công nghệ Nông nghiệp Việt Nam, 2(87), $17-22$.

Dwivedi, Y. K., Rana, N. P., Jeyaraj, A., Clement, M., \& Williams, M. D. (2019). Re-examining the Unified Theory of Acceptance and Use of Technology (UTAUT): Towards a revised theoretical model. Information Systems Frontiers, 21(3), 719-734.

Flett, R., Alpass, F., Humphries, S., Massey, C., Morriss, S., \& Long, N. (2004). The technology acceptance model and use of technology in New Zealand dairy farming. Agricultural Systems, 80(2), 199-211. doi:10.1016/j.agsy.2003.08.002

Folorunso, O., \& Ogunseye, S. (2008). Applying an enhanced technology acceptance model to knowledge management in agricultural extension services. Data Science Journal, 7(2008), 31-45. doi:10.2481/dsj.7.31

Hoai Thanh (2016). Bắt quả tang cơ sở nhúng sầu riêng vào hóa chất [Caught in the act of dipping durian in chemicals]. Retrieved January 05, 2020, from Vnexpress website: https://vnexpress.net/bat-qua-tang-co-so-nhung-sau-rieng-vao-hoa-chat-3461984.html.

Le, H. V, Le, T. H., \& Pham, T. T. P. (2012). Hiệu quả của việc xử lý Ethephon dạng đơn và kết hợp với $\mathrm{GA}_{3}, \mathrm{CaCl}_{2}$ trước khi thu hoạch đến màu sắc và phẩm chất trái cam mật (Citrus Sinensis (L.) Osbeck) [Effect of treatment of Ethephon alone and in combination with $\mathrm{GA}_{3}, \mathrm{CaCl}_{2}$ before harvest on color and quality of honey orange (Citrus Sinensis (L.) Osbeck)]. Tạp chi Khoa hoc - Trương Đại học Cần Tho, 22a(2012), 107-114.

Martins, C., Oliveira, T., \& Popovič, A. (2014). Understanding the Internet banking adoption: A unified theory of acceptance and use of technology and perceived risk application. International Journal of Information Management, 34(1), 1-13. doi:10.1016/j.ijinfomgt.2013.06.002

Nguyen, P. D., Nguyen, K. H., \& Angelina, N. H. L. (2018). Factors affecting mobile shopping: A Vietnamese perspective. Journal of Asian Business and Economic Studies, 25(2), 186205. doi:10.1108/JABES-05-2018-0012

Nguyen, T. D. (2011). Phương pháp nghiên cúu khoa học trong kinh doanh [Scientific research methods in business]. Hanoi, Vietnam: NXB Lao động Xã hội.

Nguyen, T. T. T., \& Nguyen, N. V. (2017). Các yếu tố ảnh hwởng đến việc không sủ dụng chất cấm trong chăn nuôi heo tại huyện Tân Phuớc - Tỉnh Tiền Giang [Factors affecting the non-use of banned substances in pig production in Tan Phuoc district, Tien Giang Province] (Master's thesis). Nong Lam University, Ho Chi Minh City, Vietnam.

Obiero, K., Waidbacher, H., Nyawanda, B., Munguti, J., Manyala, J., \& Arara, B. K. (2019). Predicting uptake of aquaculture technologies among smallholder fish farmers in Kenya. Aquaculture International, 27(3), 1689-1707. doi:10.1007/s10499-019-00423-0 
Phuong Hoa (2016). Liều lượng gây độc của hóa chất biến trái xanh thành chín [The toxic dose of the chemical turns green fruit into ripe]. Retrieved January 05, 2020, from Vnexpress website: https://vnexpress.net/lieu-luong-gay-doc-cua-hoa-chat-bien-trai-xanh-thanh-chin3405172.html

Rodríguez, E. T., \& Trujillo, C. E. (2013). Online drivers of consumer purchase of website airline tickets. Journal of Air Transport Management, 32(2013), 58-64.

doi:10.1016/j.jairtraman.2013.06.018

Thanh Bac \& Hoai Thuong (2013). Doanh nhân Trung Quốc thuê người tẩm hóa chất vào sầu riêng [Chinese businessman hires people to apply chemicals to durian]. Retrieved January 05, 2020, from Tuổi trẻ Online website: https://tuoitre.vn/doanh-nhan-trung-quoc-thuenguoi-tam-hoa-chat-vao-sau-rieng-584259.htm

Thuc Vy (2015). Thực hư về chất làm chín trái cây Ethephon gây hoang mang [The truth about Ethephon's fruit ripening agent causes confusion]. Retrieved January 05, 2020, from Báo Điện tử Tài nguyên \& Môi trường website: https://baotainguyenmoitruong.vn/thuc-hu-vechat-lam-chin-trai-cay-ethephon-gay-hoang-mang-281714.html

Tran, P. H. (2020). Ethephon - Chất điều hòa sinh trưởng thế hệ mới nhất [Ethephon - The latest generation growth regulator]. Retrieved March 20, 2020, from http://www.phanbonla.vn/ index.php/th-vin-nha-nong/88-ethephon-chat-dieu-hoa-sinh-truong-the-he-moi-nhat

Trung Tan (2015). Bắt quả tang ba cơ sở nhúng sầu riêng vào hóa chất làm chín [Caught redhanded of three establishments dipping durians in ripening chemicals]. Retrieved January 05, 2020, from Tuổi trẻ Online website: https://tuoitre.vn/bat-qua-tang-ba-co-so-nhungsau-rieng-vao-hoa-chat-lam-chin-965055.htm

Venkatesh, V., Morris, M. G., Davis, G. B., \& Davis, F. D. (2003). User acceptance of information technology: Toward a unified view. MIS Quarterly, 27(3), 425-478.

Venkatesh, V., Thong, J. Y. L., \& Xu, X. (2012). Consumer acceptance and use of information technology: Extending the unified theory of acceptance and use of technology. MIS Quarterly, 36(1), 157-178. doi:10.2307/41410412

Vu, D. T., \& Vo, D. T. (2011). Hiệu quả sản xuất sầu riêng tại tỉnh Tiền Giang [Efficiency of durian production in Tien Giang Province]. Tạp chí khoa hoc - Truờng Đại hoc Cần Tho, 20b(2011), 237-247.

Xuan Ngoc (2014). Hóa chất thúc chín trái cây độc cỡ nào? [How toxic is the chemical to promote fruit ripening?] Retrieved January 05, 2020, from Báo điện tử Dân trí website: https://dantri.com.vn/xa-hoi/hoa-chat-thuc-chin-trai-cay-doc-co-nao-1420313805.htm

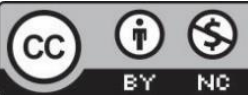

Creative Commons Attribution-NonCommercial 4.0 International License. 